MATEC Web of Conferences 32, 02003 (2015)

DOI: $10.1051 /$ matecconf $/ 20153202003$

C) Owned by the authors, published by EDP Sciences, 2015

\title{
3D electrostatic actuator fabricated by non-ablative femtosecond laser exposure and chemical etching
}

\author{
Tao Yang ${ }^{1, \text { a }}$ and Yves Bellouard ${ }^{1}$ \\ ${ }^{1}$ Galatea Laboratory, IMT/STI, Ecole Polytechnique Fédérale de Lausanne (EPFL) \\ Rue de la Maladière 71b, 2002 Neuchâtel, Switzerland
}

\begin{abstract}
We demonstrate the novel design of an electrostatic micro-actuator based on monolithic three-dimensional (3D) shapes fabricated by non-ablative femtosecond laser exposure combined with chemical etching. Further, we present a single-scan stacking approach exploited in the fabrication of the 3D actuator to create crack-free, high contrast, high fidelity and integrated micro-structures. Influential parameters: energy per pulse, polarization, scanning spacing and stacking direction were systematically studied to predict and control the etching rate of 3D planes. Finally, we report the characterization of the actuator and its potential application in optomechanics to show a complete scenario of femtosecond laser machined integrated 3D micro-systems incorporating multiple functionalities.
\end{abstract}

\section{Introduction}

An actuator is a type of device that converts its operating energy source into motion and/ or force in a controllable and preferably, in a reversible way. At the micro-scale, actuators have been extensively investigated and are essential part of a sub-class of Micro-Electro-Mechanical Systems (MEMS). In optomechanics and adaptive optics, devices like deformable mirrors and interferometers, require micro-actuation for fine adjustment and positioning that can be compact and operated in the three dimensions. However and essentially due to the lack of suitable manufacturing processes, micro-actuators remain essentially planar devices; furthermore, they often require cumbersome fabrication process involving multiple steps that reduce their integration potential into more compact and more cost-effective ensemble.

Among fabrication techniques, femtosecond laser micro-machining and subsequent chemical etching, using aqueous solution of hydrofluoric acid (HF), has shown its great potential in fabricating monolithic $3 \mathrm{D}$ devices that combine multiple functions, like fluidic, mechanical and optical functions [1]. It could also open up the third dimension for existing actuation principles for development of integrated 3D micro-actuators.

We address the gap from two dimensional (2D) assembly to $3 \mathrm{D}$ monolith by investigating a monolithic $3 \mathrm{D}$ electrostatic actuator fabricated by femtosecond laser irradiation and HF etching. Non-uniform electric field induces an effective dipole moment in dielectrics and also exerts a net electrostatic force on them. This electrostatic interaction due to the non-uniformity of the field is termed as dielectrophoresis (DEP) [2]. A roadblock to DEP implementation is the need for non-trivial shapes, eventually $3 \mathrm{D}$ ones, for creating non-uniform electric field. Benefitting from 3D manufacturing capability of femtosecond laser, we designed a DEP actuator of nontrivial shapes out of a single piece fused silica (amorphous $\mathrm{S}_{\mathrm{i}} \mathrm{O}_{2}$ ) substrate.

During fabrication of micro-structures like buried cantilevers, vertical walls and slopes using multiple-scan stacking configuration, higher stress is accumulated at the corner of patterns. As a consequence, stress induced cracks could take place even using a laser with a very low energy per pulse [3]. Delicate micro-components, like slender cantilevers and hinge joints, are more susceptible to breakage induced by cracks. For integrated complex fabrication, synchronized etching of structures of different dimensions and different accessibility to etchant is also very critical to obtain the desired final shapes. To preserve the shape and get a higher yield rate, we developed a single-scan stacking approach for fabrication of crack-free structures. Energy per pulse, polarization, scanning spacing and stacking direction were systematically studied to predict and control the etching rate of 3D planes, aiming for high contrast and high fidelity structures.

Taking advantage of the patterning strategy developed, we managed to fabricate crack-free structures of good shape control for DEP actuation. The general characterization of the designed DEP actuator is presented to demonstrate the possibility of femtosecond laser machined multi-functional micro-systems.

\section{DEP actuator: design and fabrication}

\subsection{Working principle and design}

${ }^{a}$ Corresponding author: tao.yang@epfl.ch

This is an Open Access article distributed under the terms of the Creative Commons Attribution License 4.0, which permits unrestricted use, distribution, and reproduction in any medium, provided the original work is properly cited. 
As a proof-of-concept, we investigated a cantilever moving in a non-uniform electric field created by a Vshape arrangement of electrodes. This concept was inspired from R. Jebens and co-workers [4] who suggested the idea of positioning an optical fiber.

The working principle is briefly outlined in Fig. 1. When an external driving voltage is applied on both sides of the V-shape groove, a non-uniform electric field rises as indicated by the field lines in Fig. 1(b). The presence of the electrostatic field causes the appearance of an effective dipole moment in the cantilever. Since the electrostatic field is non-uniform, a net force rises distributed along the cantilever causing the slim structure to bend.
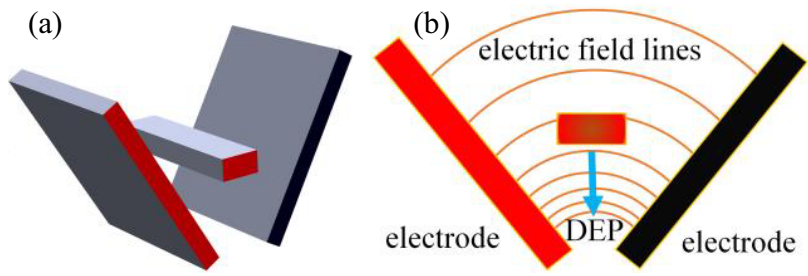

Fig. 1 (a) 3D rendering of the V-shape groove and the cantilever. (b) Dielectrophoresis actuation of the cantilever.

We generalized this concept and demonstrated it on a monolithic design. The actuator includes a buried cantilever with a high aspect ratio of 1: 300 symmetrically placed in a V-shape groove fabricated out of a single piece of a $500 \mu \mathrm{m}$-thick fused silica substrate by femtosecond laser exposure and HF etching [5]. A diagram of the monolithic design is shown in Fig. 2.

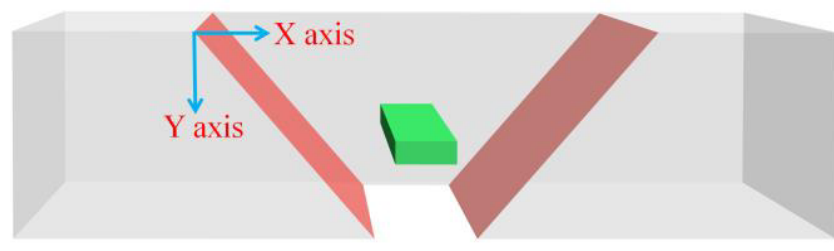

Fig. 2 Diagram of the monolithic DEP actuator.

Both sides of the V-shape groove are coated with conductive layer, e.g. gold or indium tin oxide (ITO) for high transparency purpose, serving as electrodes. Before depositing the conductive layers, the machined pattern is partly covered by a masking tape. The mask is carefully designed to prevent bridging both sides of the V-shape groove and to insulate electrically the cantilever from either side of the groove.

\subsection{Fabrication}

An overview of the fabrication processing is as followed. Laser exposure is provided by an ytterbium-fiber amplifier laser with pulse duration of 270 femtoseconds. The laser emits pulses of $230 \mathrm{~nJ}$ energy per pulse at a frequency of $800 \mathrm{kHz}$, corresponding to an average power of $184 \mathrm{~mW}$. The laser beam is focused down to a beam waist about $1.5 \mu \mathrm{m}$ using an objective with an effective numerical aperture (NA) of 0.4. The substrate is a $500 \mu \mathrm{m}$-thick fused silica. The laser beam is focused inside fused silica to an intensity below the ablation threshold.

The specimen is loaded onto a dual axes motorized stage which enables plane motion, whereas the laser focal spot is translated by a $\mathrm{Z}$-axis stage. Those three axes can achieve synchronized motion controlled by predefined programs, thus achieving 3D-scanning of the laser focal point. Since nonlinear absorption takes place, material properties can be locally modified in the three dimensions and in particular below the surface of transparent materials. Following the laser modification step, the specimen is then immersed into a $2.5 \%$ aqueous solution of HF bath for pattern etching while keeping low tapered profile.

Laser tracks are scanned layer by layer from bottom to top. A moderate NA of 0.4 and a relative thinner substrate of $500 \mu \mathrm{m}$ avoid severe spherical aberration induced by dielectric air interface causing deterioration of the laser focus and consequent glass modification distortion [6].

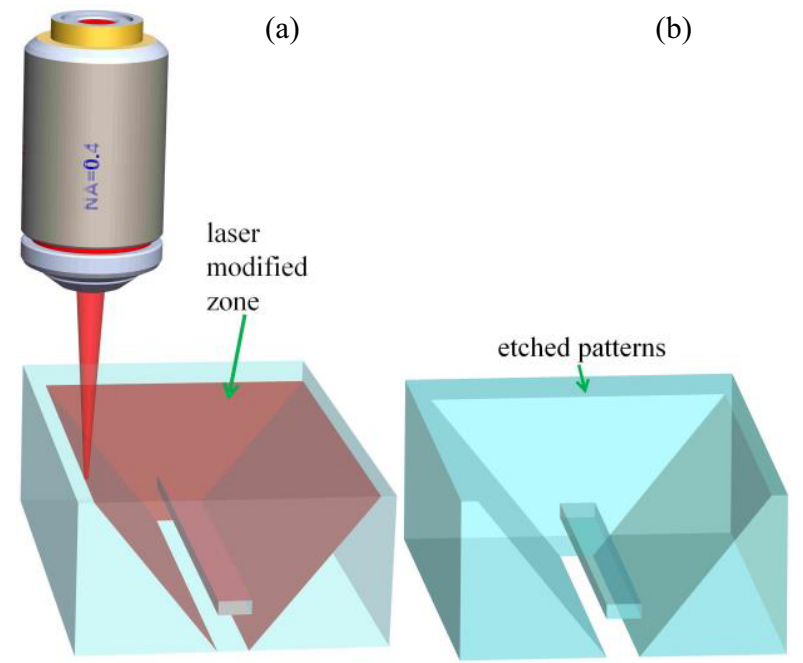

Fig. 3 Illustration of the two steps of femtosecond laser processing combined with chemical etching. (a) First, the substrate is modified by a tightly forced femtosecond laser emitting low energy pulse, no ablation takes place. (b) The second step is to immerse the substrate into preferential chemical etching and to reveal the patterns.

Multiple-scan stacking configuration with fixed pitch of 4 to $6 \mu \mathrm{m}$ was implemented to define the contour of the $\mathrm{V}$-shape groove, the vertical side wall and the surroundings of the buried cantilever. Additional lines as access ports from surfaces to buried structures were written to extend the exposure length to the etchant. It sounds intuitively that multiple-scan and smaller pitches would increase the interconnectivity of adjacent laser affected zones and result in an accelerated etching rate. However, we will show in next section that single-scan and bigger pitches can increase the etching rate for certain patterns and polarizations. Furthermore, one issue with multiple-scan stacking is that the induced internal stress [7] surrounding the laser affected zones would be much higher especially for patterns with sharp corners, increasing the risk of spontaneous cracking and damaging 
delicate structures. Fig. 4 shows stress induced cracks after machining.
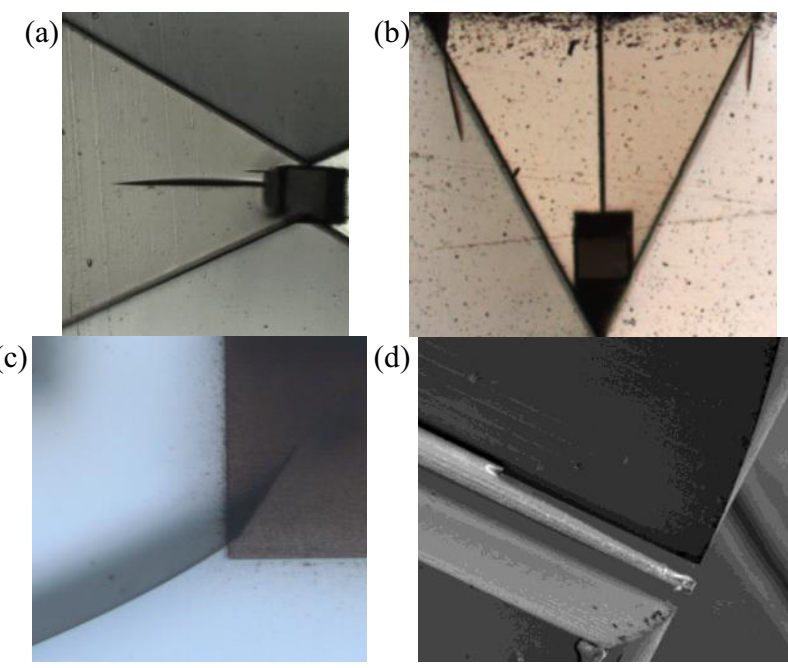

Fig. 4 (a)-(c) Camera images of crack propagation due to multiple-scan. (d) Scanning Electron Microscopy (SEM) image of a broken cantilever tip due to cracks.

For integrated fabrication combining bulky structures and tiny features, crack-free structures of high contrast are essential.

Although the etching rate results for single laser track has been well presented [8], the etching rate of stacking patterns with changeable pitches is yet to be demonstrated. Fixed pitch results in uniform etched length for the identical pattern, however it appears to be inflexible for etching of patterns of different dimensions and different accessibility to the etchant. For integrated fabrication, synchronized etching of different features is also important to obtain desired shapes.

\section{Single-scan stacking strategy}

Instead of multiple-scan, we use a single-scan stacking strategy and demonstrate its benefit of crack-free fabrication. Three plane configurations formed by stacking laser tracks along different orientations as shown in Fig. 5 are studied. Energy per pulse, polarization and scanning spacing were systematically studied to predict and control the etching rate of individual 3D plane.
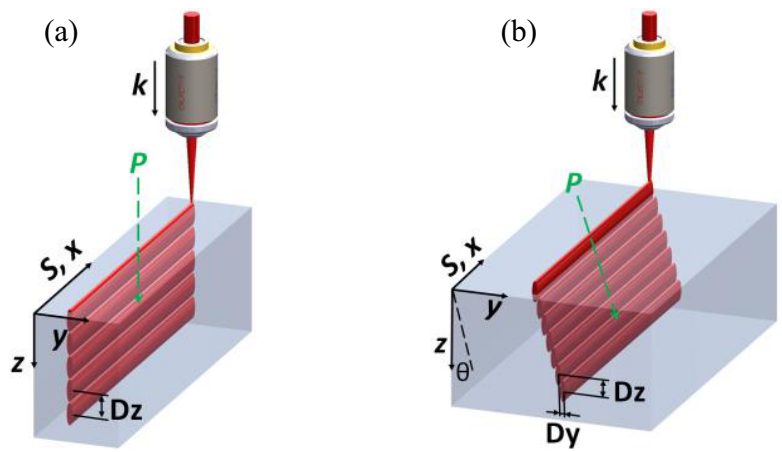

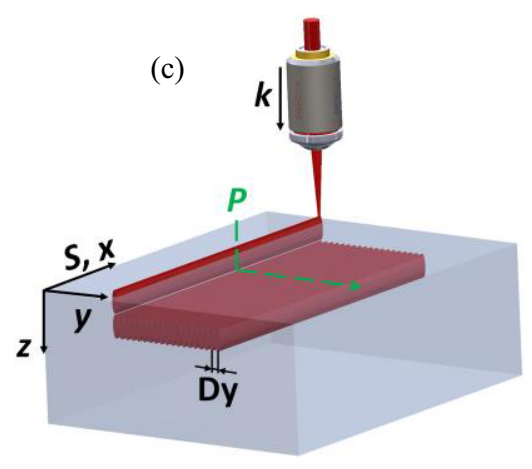

Fig. 5 Single-scan stacking of three fundamental configurations of $3 \mathrm{D}$ planes. $\mathrm{S}$ and $\mathrm{k}$ denote the laser scanning direction and the propagation direction, respectively. $\mathrm{x}, \mathrm{y}$ and $\mathrm{z}$ are coordinate axes. $\mathrm{P}$ is the etchant flow direction. (a) XZ plane: Dz terms the $Z$-spacing between two adjacent lines. (b) XYZ plane: Dz and Dy are the spacing projected on $\mathrm{Z}$-axis and $\mathrm{Y}$-axis, respectively. The total spacing is calculated as mean square root of Dz and Dy. $\theta$ is defined as the angle of the orientation of the plane with respect to vertical $\mathrm{Z}$ direction. (c) XY plane: Dy denotes the spacing between two adjacent parallel lines.

\subsection{Etching rate of 3D planes}

The HF gets access to the XZ plane from the surface and flows transversely across the laser tracks as shown in Fig. 5(a). The spacing Dz between two adjacent tracks ranges from 3 to $24 \mu \mathrm{m}$ and both perpendicular and parallel polarization with respect to the laser scanning direction $\mathrm{S}$ are used. After etching for 4 hours, the etching rate measured at different positions of the boundary between the etched and un-etched laser lines is averaged and plotted in Fig. 6.
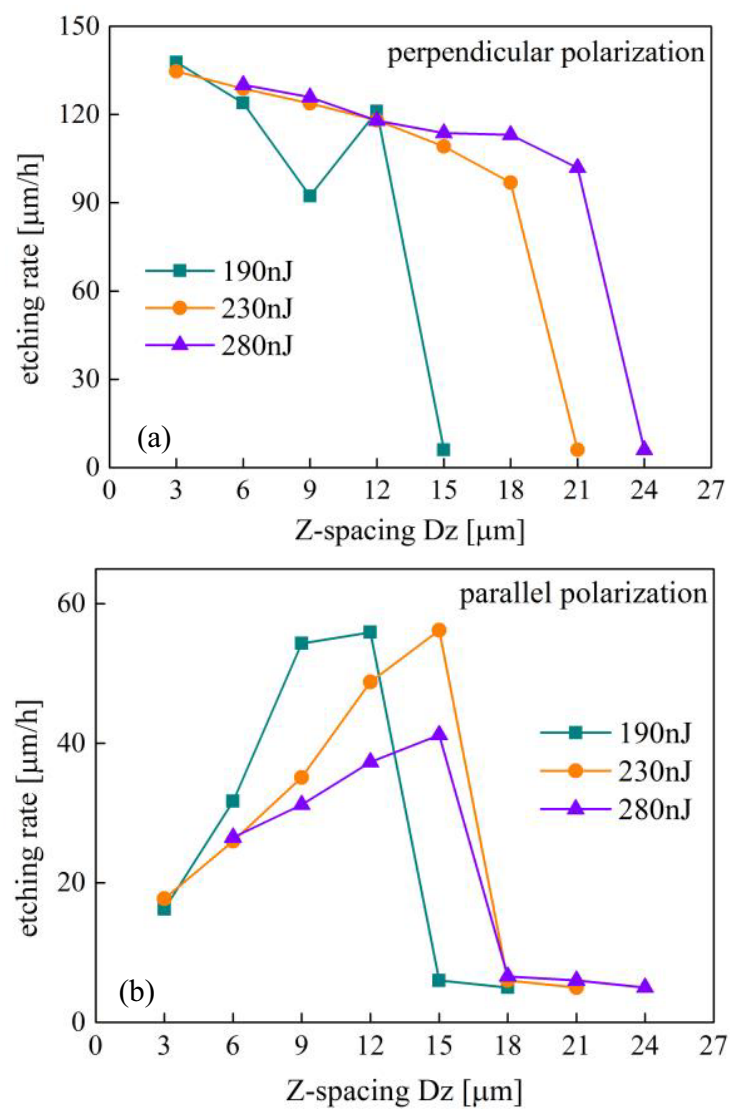
Fig. 6 Averaged etching rate of XZ plane at spacing ranging from 3 to $24 \mu \mathrm{m}$. Energy per pulse is set to 190,230 and $280 \mathrm{~nJ}$. (a) Perpendicular polarization. (b) Parallel polarization.

It may sound intuitive to use smaller spacing to get an accelerated etching rate. On the contrary, bigger spacing can remarkably increase the etching rate for $\mathrm{XZ}$ planes using parallel polarization as shown in Fig. 6(b). Compared to parallel polarization, perpendicular polarization results in much faster etching rate up to 140 $\mu \mathrm{m} / \mathrm{h}$ and shows opposite trend as the pitch increases. There exists a spacing threshold, above which etching process dramatically slows down to below $10 \mu \mathrm{m} / \mathrm{h}$. The thresholds for 190 and $280 \mathrm{~nJ}$ energy per pulse are 12 and $21 \mu \mathrm{m}$ using perpendicular polarization, respectively. Increasing the energy per pulse can reduce the laser track numbers and shorten the machining time. Considering that nanogratings are found in the exposure regime considered here and the length of the nanograting region follows a logarithmic trend as the energy per pulse increases, they suggest the interconnectivity of nanograting planes determines the size of the spacing threshold.

V-shape grooves are basically a combination of inclined walls and vertical walls. To verify the possibility of non-trivial shape machining using single-scan stacking, inclined walls as shown in Fig. 5(b) are machined at a fixed energy per pulse of $215 \mathrm{~nJ}$. The ratio of Dz to Dy is set to $1: 1,2: 1$ and $1: 2$, resulting in an inclination angle $\theta$ at 45,27 and 63 degrees, respectively. The spacing projected along $\mathrm{z}$ direction is set to $0.1,0.2,0.3,0.4,0.6$, $0.8,1,1.2,1.6$ and $2 \mu \mathrm{m}$. The results of the averaged etching rate are shown in Fig. 7.
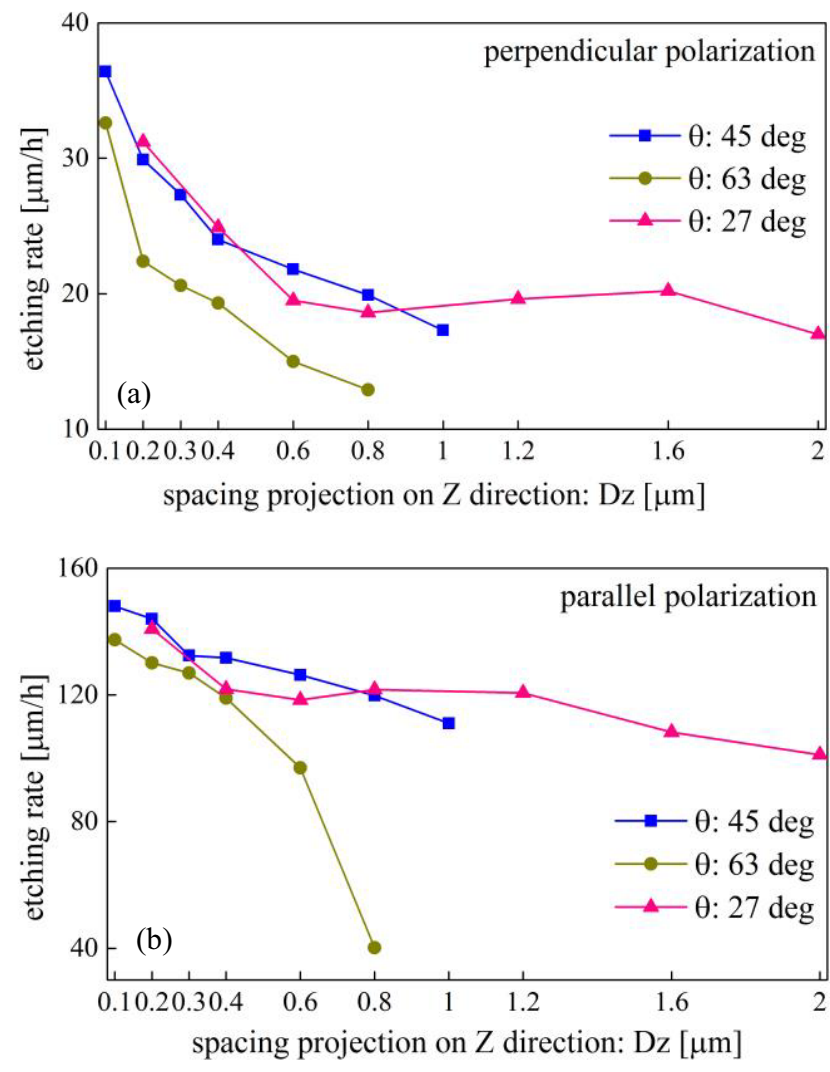

Fig. 7 Averaged etching rate of $X Y Z$ plane. $X$ axis is set as the spacing projection on $Z$ direction for comparison among different inclinations. Energy per pulse is fixed at 215 nJ. (a) Perpendicular polarization. (b) Parallel polarization.

Parallel polarization is much more efficient in etching of XYZ plane than perpendicular polarization. The results in above two cases suggest a solution for fast etching of V-shape grooves. When scanning laser beam layer by layer defining the contour of the two slopes and the vertical side wall together in Fig. 3, the polarization should be kept constant: parallel to lines defining the slope. When scanning turns 90 degrees at the corners, the polarization is changed automatically to perpendicular to lines defining the vertical wall. However, when the two slopes become vertical walls, polarization should always be perpendicular to the scanned lines. This is very practical and important.

In-plane planes shown in Fig. 5(c) are machined 30 $\mu \mathrm{m}$ below the top surface. An access port formed by a few Z-stacking laser tracks is used for HF flow. The lateral spacing is from minimum $0.1 \mu \mathrm{m}$ to maximum 2 $\mu \mathrm{m}$ with insertions at $0.2,0.3,0.6,1,1.2$ and $1.5 \mu \mathrm{m}$. The averaged etching rate by measuring the etched lengths at different positions is shown in Fig. 8.
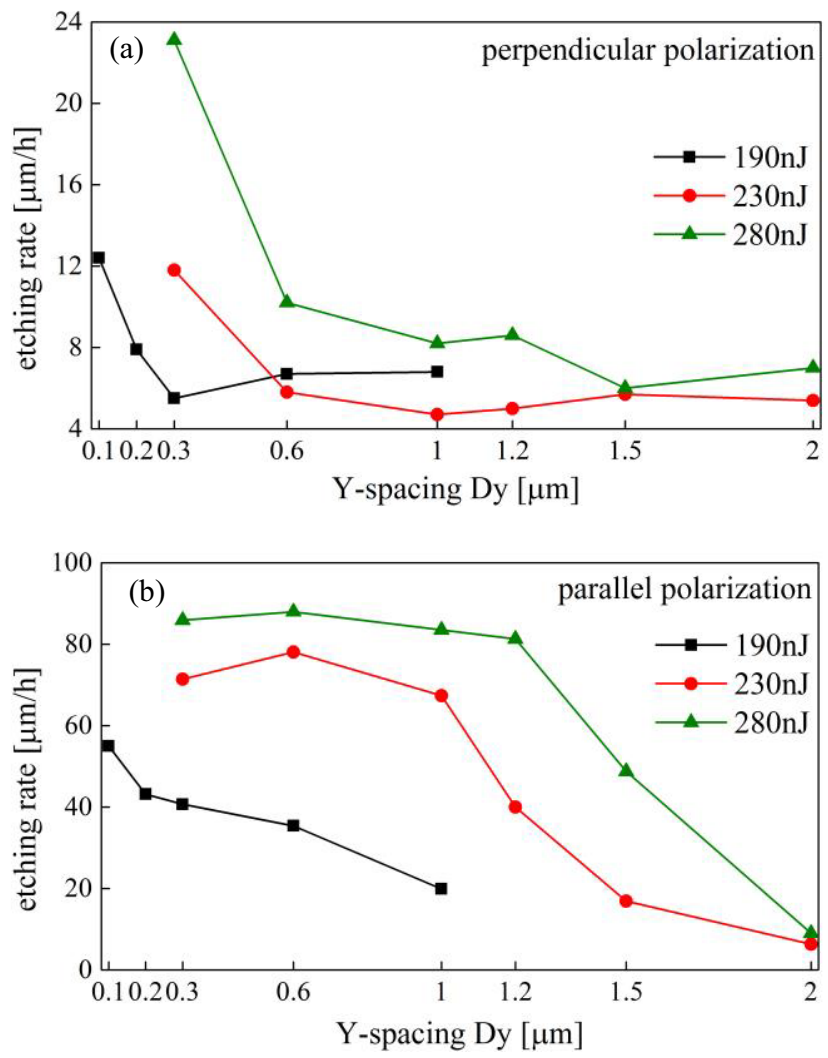

Fig. 8 Averaged etching rate of XY plane. Energy per pulse is 190, 230 and $280 \mathrm{~nJ}$. (a) Perpendicular polarization. (b) Parallel polarization.

Parallel polarization machining is faster $(5 \times$ folds $)$ in etching rate compared to perpendicular polarization machining. Similar to the data of etching XZ planes, there seems also exist a spacing threshold, above which etching slows down. We also suspect the 
interconnectivity influenced by nanograting size determines the etching rate. For parallel polarization machined XY planes using smaller spacing, since the nanogratings orient normal to the writing direction, the periodic nano-slots in individual laser tracks have more chance to align to the nano-slots in adjacent lines, forming a channel for etchant flow; however, when using perpendicular polarization, the nanogratings align with the writing direction, the etchant will encounter alternative layers separated by high density materials, resulting in reduced cumulative etching rate even using very small spacing as shown in Fig. 8(a).

\subsection{Discussion}

SEM images reveal the morphology of the successively written laser tracks. Nanogratings are embedded inside the laser affected zone, forming an ellipse-like envelope along the beam propagation direction [9]. The periodic gratings, depending on laser polarization and energy per pulse, change their size and orientation. Moreover, the spacing and the stacking direction also influence the overall connection of gratings. Fig. 9 shows a comparison of the morphologies between a Z-stacking vertical wall and a 45 degree slope wall.
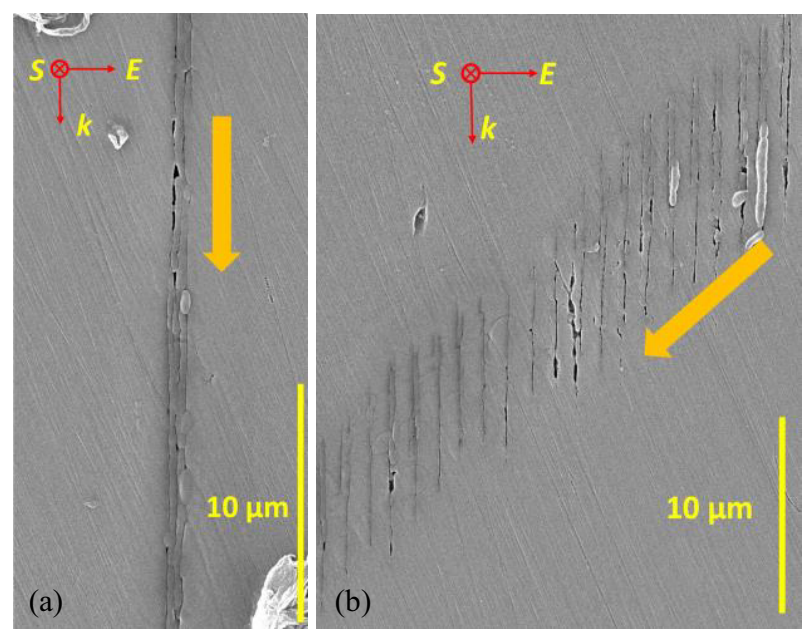

Fig. 9 SEM images of cross sectional cut of a Z-stacking vertical wall (a) and a 45 degree slope wall (b). Bold arrows in both images show the etching direction.

For the vertical walls, the nanogratings seem to be self-aligned to each other, forming a long channel for etchant flow. Whereas for the slopes, periodic blocks are encountered in the course of etching, slowing down the etching rate. This particular case reveals the principles behind the non-trivial etching of $3 \mathrm{D}$ planes: the interconnectivity of nanogratings plays a key role.

\section{Characterization of DEP actuator}

Exploiting the single-scan stacking strategy, crack-free and fast etched structures for DEP actuation is fabricated. No cracks are found before and after etching process.
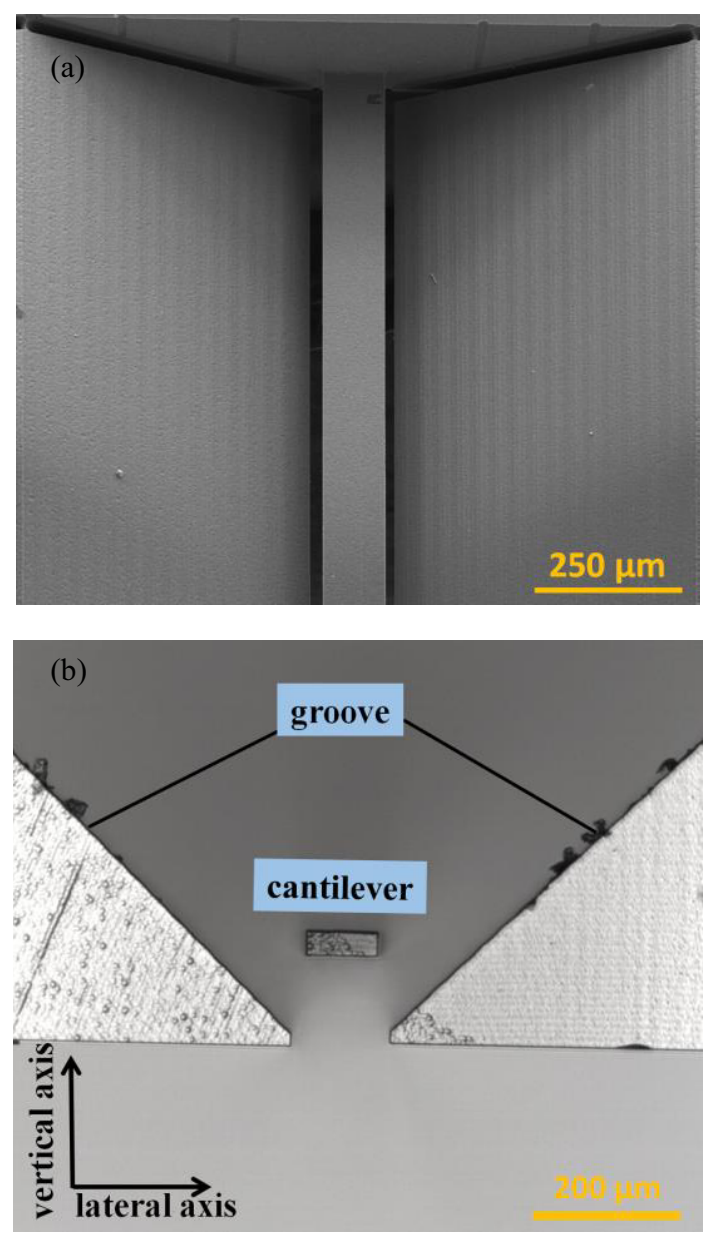

Fig. 10 SEM images of the designed DEP actuator. (a) Top view. (b) Size view.

Performances of the DEP actuator are examined by measuring the deflection of the cantilever tip by a camera system and a positioning sensing detector (PSD).

After applying a direct driving voltage, the cantilever shows stable deflection towards high electric field near groove bottom. A range of $20 \mu \mathrm{m}$ is well attainable when applying a moderate magnitude of voltage. The calculated DEP force has a density in the order of a few $\mu \mathrm{N} / \mathrm{mm}^{3}$ on the cantilever.

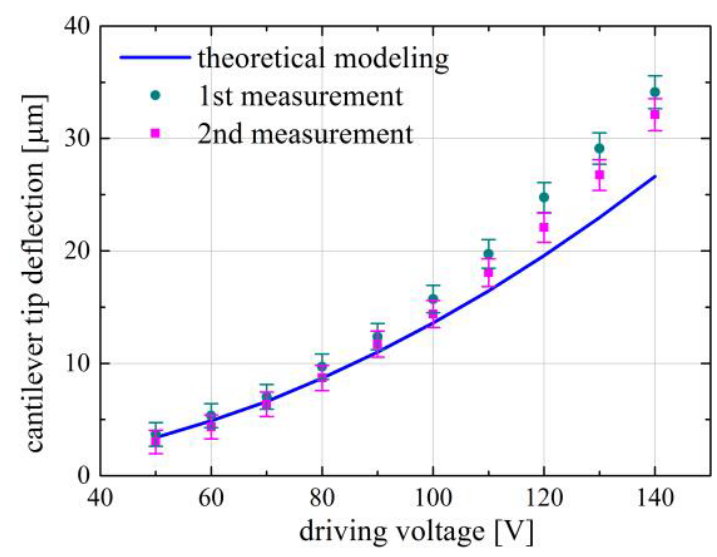

Fig. 11 Experimental results and theoretical analysis of the cantilever deflection under direct voltage. 
Both direct voltage and alternating voltage are applied to excite the vibration of the cantilever. The first four resonances along vertical direction as indicated in Fig. 10 (b) are measured to be at 200,1262, 3525 and $6995 \mathrm{~Hz}$, respectively.

The time domain transient response is also measured using impulses and step inputs. The damping ratio is evaluated to be 0.013 and the quality factor is about 40 . We measure an averaged settling time of $200 \mathrm{~ms}$ for a falling edge input from $60 \mathrm{~V}$ to $0 \mathrm{~V}$.

Because of the high transparency of fused silica over a wide range of light wavelength, our designed DEP actuator is also promising in optical applications. The cantilever itself can act as a waveguide, thus working as an optical switch when actuated. This approach was adopted in the PSD system for measuring the deflection of the cantilever.

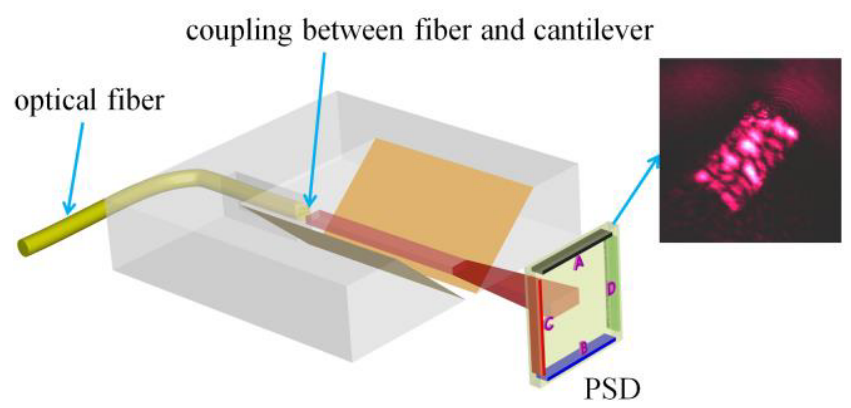

Fig. 12 Demonstration of an optical coupling between the cantilever and an optical fiber. The fiber is guided by a machined trench whose width is about the diameter of the fiber. The trench is monolithically fabricated together with the actuator, enabling accurate alignment between the fiber and the cantilever. An effective butt coupling between them initiates the transmission of a Helium-Neon laser beam through the cantilever.

\section{Conclusion}

The novelty of our work lies in the introduction of a single-scan stacking approach instead of multiple scanning for designing crack-free integrated 3D microstructures using femtosecond laser machining and chemical etching. The shape fidelity of the 3D design of a DEP actuator was improved taking advantage of our machining strategy. This approach could be a practical guidance of fabricating more sophisticated microstructures in various fields.

\section{Acknowledgments}

This work was supported by the European Research Council (ERC) through its starting grant Galatea. The Galatea Laboratory in EPFL acknowledges the sponsoring of the Richemont Group.

\section{References}

[1] Y. Bellouard, A. A. Said and P. Bado, Opt. Exp. 13, 6635 (2005).
[2] H. A. Pohl, J. Appl. Phys. 22, 869 (1951).

[3] S. Ho, P. R. Herman and S. Aitchison, Appl. Phys. A 106, 5 (2012).

[ 4 ] R. Jebens, W. Trimmer and J. Walker, Sens. Actuators 20, 65 (1989).

[5] T. Yang and Y. Bellouard, in CLEO: 2014, (2014) AW1H. 5.

[6] C. Hnatovsky, R. S. Taylor, E. Simova, P. P. Rajeev, D. M. Rayner, V. R. Bhardwaj and P. B. Corkum, Appl. Phys. A 84, 47 (2006).

[7] A. Champion, M. Beresna, P. Kazansky and Y. Bellouard, Opt. Exp. 21, 24942 (2013).

[8 ] C. Hnatovsky, R. S. Taylor, E. Simova, V. R. Bhardwaj, D. M. Rayner and P. B. Corkum, Opt. Lett. 30, 1867 (2005).

[9] Y. Bellouard, E. Barthel, A. A. Said, M. Dugan and P. Bado, Opt. Exp. 16, 19520 (2008). 